\title{
Caséine K, substrat de la chymosine et comparaison entre les phénomènes de coagulation du lait et du sang
}

\author{
par \\ P. JOLLES*
}

Pour des raisons de "copyright " nous ne pouvons publier ici que le résumé de cette intéressante conférence, car certaines parties sont en cours de publication:

- d'une part, sous forme d'un article sous presse dans «Trends in Biochemical Science " (TIBS), 7 (1982).

"Comparison between the clotting of blood and milk";

- d'autre part, comme chapitre dans l'ouvrage "Evolution des Protéines ", Masson Ed. (1982).

"Comparative structure features between the blood and milk clotting processes.

Nous prions nos lecteurs de bien vouloir nous en excuser.

Les deux phénomènes de coagulation les plus étudiés sont ceux du lait et du sang. Les deux protagonistes de la coagulation du lait sont l'enzyme appelée chymosine et le substrat dénommé caséine $\mathrm{K}$. La dernière étape du processus de coagulation du sang met en jeu le fibrinogène (substrat) et la thrombine (enzyme).

Cet exposé est, dans sa première partie, centré sur la caséine $\mathrm{K}$ de vache : sa séquence, la structure développée et la localisation de ses groupements prosthétiques sucrés ainsi que sa structure secondaire seront discutées. Un tour d'horizon est ensuite consacré à la caséine $\mathrm{K}$ de colostrum puis à des caséines $\mathrm{K}$ d'autres origines,

* Laboratoires des Protéines, Université de Paris V. 
notamment à celle d'origine humaine qui est extrêmement riche en sucres.

La deuxième partie de la conférence est consacrée à une comparaison au niveau moléculaire des deux phénomènes de coagulation qui semblent présenter un grand nombre de points communs. Une homologie structurale a été mise en évidence entre la caséine $\mathrm{K}$ et la chaîne $\gamma$ du fibrinogène, permettant de suggérer une possible relation phylogénétique entre les chaînes $B \beta$ et $\gamma$ du fibrinogène et les caséines K. Par ailleurs, il a pu être montré que les acides aminés impliqués dans les interactions K-caséine/chymosine et fibrinogène/ thrombine sont les mêmes. 